\title{
The Inhibitory Action of Fatty Acids on the Growth of Escherichia coli
}

\author{
By J. P. FAY AND R. N. FARIAS \\ Instituto de Química Biológica, Facultad de Bioquímica, Química y Farmacia, \\ Universidad Nacional de Tucumán, Chacabuco 461, San Miguel de Tucumán, Argentina
}

(Received 3 January 1975; revised 25 June 1975)

\begin{abstract}
SUMMAR Y
The effect of fatty acids on Escherichia coli KI2 was dependent on the source of the inoculum, the growth phase and the washing of the bacteria. The effects of saturated fatty acids from $\mathrm{C}_{4}$ to $\mathrm{C}_{\mathrm{I}} 6$ and oleic acid at two concentrations ( $0 \cdot \mathrm{I}$ and $0.4 \%, \mathrm{w} / \mathrm{v}$ ) were determined on $E$. coli $\mathrm{KI} / \mathrm{I} 54$ growing exponentially in five different culture media. Depending on the media, $0.1 \%$ fatty acids increased the doubling times of the cultures by up to $96 \%$. Fatty acids of medium chain length (C6 to $\mathrm{CII}$ ) at $0.4 \%$ produced a decrease in cell concentration, nonanoic and decanoic acids being the most effective. A correlation was found between the decrease in cell concentration and the loss of viability of the cultures after addition of $0.4 \%$ decanoic acid, with stationary-phase bacteria being affected more than those from exponential-phase cultures. Experiments carried out with $E$. coli B and $\mathrm{C}$ gave results similar to those obtained with $E$. coli $\mathrm{KI} 2 / \mathrm{I} 54$.
\end{abstract}

\section{INTRODUCTION}

The growth of both Gram-positive and, to a lesser extent, Gram-negative bacteria is inhibited by fatty acids (Eisler \& Von Metz, I 968; Freese, Sheu \& Galliers, I973; Salanitro \& Wegener, 197I; Sheu \& Freese, 1972; Sheu, Konings \& Freese, 1972; Weeks et al. 1969).

Escherichia coli has been used in a number of studies on the effects of fatty acids on the growth of Gram-negative bacteria, but the results obtained have not been consistent. For example, although increased inhibition was found with increasing chain length from C6 to $\mathrm{C}_{9}, \mathrm{Cr}$ o and $\mathrm{C}$ I $\mathrm{I}$ acids had less effect than $\mathrm{C} 9$ (Salanitro \& Wegener, I97I). In addition to the effects of chain length, the growth medium also appears to influence results; Weeks et al. (1969) reported an increase in generation time when $\mathrm{C} 4, \mathrm{C} 6$ or $\mathrm{C} 8$ acids were added at a concentration of $0.1 \%$ to a medium containing amino acids, but Salanitro \& Wegener (I97I) found no inhibition with $\mathrm{C}_{4}$ and $\mathrm{C}_{5}$ acids added to an acetate minimal medium. Lauric acid ( $0.1 \%)$ inhibits growth for at least $10 \mathrm{~h}$ when added to a glucose minimal medium (Kato \& Arima, I97I) but apparently has no effect in a minimal medium containing acetate (Salinitro \& Wegener, I97I).

The effects of fatty acids on bacteria were not always studied at the same stage of growth and results may also have been influenced by the use of different strains of $E$. coli as the test organism. To obviate these difficulties, we used strain KI2/I54 (chosen because it could allow future genetical analyses taking advantage of its $\mathrm{Hfr}$ properties) to investigate the effects of chain length of acid, and medium, on the growth of $E$. coli. We found that fatty acids added to cultures at the same stage of growth and containing the same concentration 
of bacteria may produce different inhibitory effects depending on the chain length and concentration of the acid, but that the stages of growth and the source of the inoculum can also influence the results.

\section{METHODS}

Organisms. Escherichia coli $\mathrm{KI} 2 / \mathrm{I} 54$ is a thiamine-requiring $\mathrm{Hfr}$ derivative of strain $\mathrm{KI} 2$ AT III A (Nagel de Zwaig, Antón \& Puig, 1962) with resistance to colicins V and I. Escherichia coli $\mathrm{B}$ and $\mathrm{C}$ are prototrophic strains.

Fatty acids. All fatty acids were used without further purification. Commercial sources were : Eastman Organic Chemicals, Rochester, New York, U.S.A. for butyric, valeric, hexanoic, heptanoic, nonanoic and decanoic acids; Sigma or Fluka Chemiske Fabrik, Buchs, Switzerland for undecanoic, lauric, tridecanoic, myristic, pentadecanoic and palmitic acids; E. Merck A.G., Darmstadt, Germany, for oleic acid. Fatty acids were classified according to their chain length as short $\left(\mathrm{C}_{4}\right.$ and $\mathrm{C}_{5}$ ), medium ( $\mathrm{C} 6$ to $\mathrm{C}_{\mathrm{I}}$ ) and long chain (from CI 2 on) (Salanitro \& Wegener, I97I).

Media and culture conditions. Cultures were grown either in nutrient broth (Oxoid) or in a synthetic medium M9 (Adams, 1959) containing, in addition to a carbon source, $1.5 \%$ (v/v) Triton X-10o (The Rohm and Haas Co., Philadelphia, U.S.A.). The detergent was present in all media and was necessary to disperse the fatty acids (when present). M9 medium contained ammonium chloride as the nitrogen source and, for growth of E. coli K12/154, thiamine ( $\mu \mathrm{g} / \mathrm{ml}$ ) was added. The $\mathrm{pH}$ was $6 \cdot 7$ to $7 \cdot 0$. The following carbon sources were added as required at $0.5 \%$ : glucose, succinate, acetate, and Bacto Casamino acids (vitamin free, Difco).

Cultures were grown at $37{ }^{\circ} \mathrm{C}$ in a New Brunswick gyratory shaker, model G-25, in $50 \mathrm{ml}$ capacity culture flasks containing $10 \mathrm{ml}$ medium. Cultures were inoculated with bacterial suspensions grown in the same media to mid-exponential phase (unless otherwise specified) at an initial turbidity of between 0.020 and 0.050 . Turbidity was monitored at 420 or $560 \mathrm{~nm}$ with a Spectronic 20 colorimeter (Bausch \& Lomb). Fatty acids (neutralized with $\mathrm{KOH}$ ) were added at $0 . \mathrm{I}$ to $0.4 \%$ final concentration. Viable counts were carried out by diluting the cells in M9 medium and by spreading them on to nutrient agar plates containing $\mathrm{I} \cdot 5 \%$ (v/v) Triton X-Ioo.

\section{RESULTS}

\section{Phases of bacterial growth and growth inhibition by fatty acids}

Using overnight cultures (presumably stationary-phase), Kato \& Arima (I97I) reported that $0.1 \%$ lauric acid produced a lag phase of $10 \mathrm{~h}$ in a glucose minimal medium. Stationaryphase cultures of $E$. coli KI2/r54 inoculated into a similar medium showed a lag of $\mathrm{I}$ to $2 \mathrm{~h}$ in the absence of added fatty acid and of 5 to $8 \mathrm{~h}$ in the presence of $0.1 \% \mathrm{C}_{4}$ to $\mathrm{C}_{3} 3$ fatty acids. When this experiment was repeated using an inoculum from a culture in mid-exponential phase, however, the addition of $0^{\circ} \mathrm{I} \%$ fatty acids of $\mathrm{C}_{7}$ to $\mathrm{C}_{13}$ or oleic acid did not increase the length of the lag phase (I to $\mathrm{I} \cdot 5 \mathrm{~h}$ ) while a lag of 3 to $8 \mathrm{~h}$ was recorded in the presence of $\mathrm{C}_{4}$ to $\mathrm{C} 6$ fatty acids. The influence of the growth phase of the inoculum on the effect of lauric acid is shown in Fig. I.

Salanitro \& Wegener (197I) reported that inhibition of growth by $5 \mathrm{~mm}$ medium-chain fatty acids occurred when mid-exponential phase bacteria growing in a succinate minimal medium were washed and transferred to a similar medium containing acetate. To avoid such changes in the medium carbon source, a mid-exponential-phase culture growing on succinate was used, without washing, as inoculum into succinate- or acetate-containing 


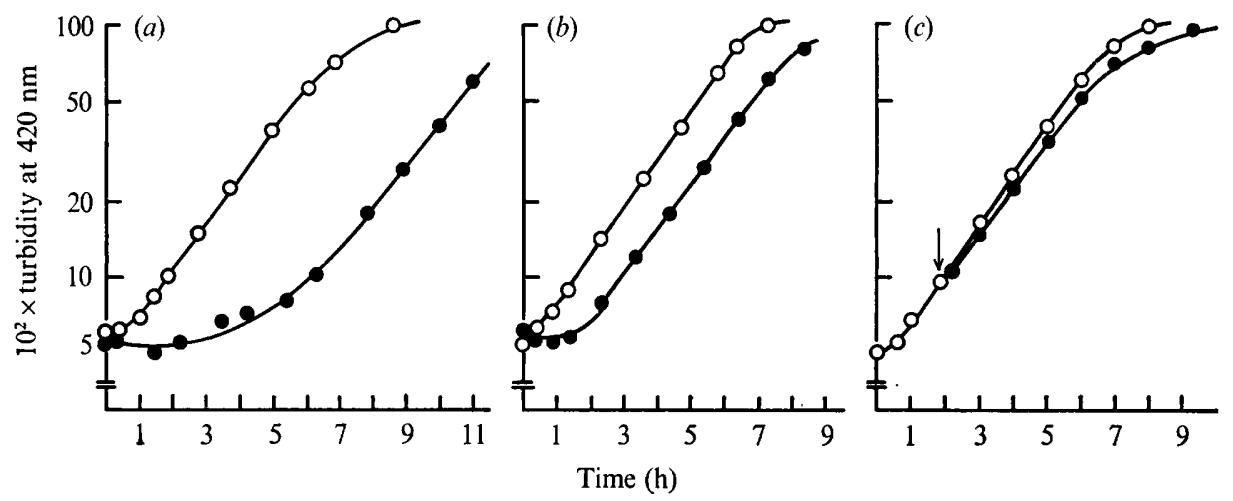

Fig. I. Effect of lauric acid on the growth of $E$. coli $\mathrm{K}$ I 2/ I 54 in a glucose minimal medium. Growth was measured as turbidity. Media were inoculated with: $(a)$ a $2 \mathrm{~h}$ stationary-phase culture, $(b)$ a mid-exponential phase culture, $(c)$ an exponentially-growing culture. In $(c) 0.1 \%$ lauric acid was added (arrow). $\bigcirc$, Glucose minimal medium; $\bigcirc$, glucose minimal medium $+0 \cdot 1 \%$ lauric acid.

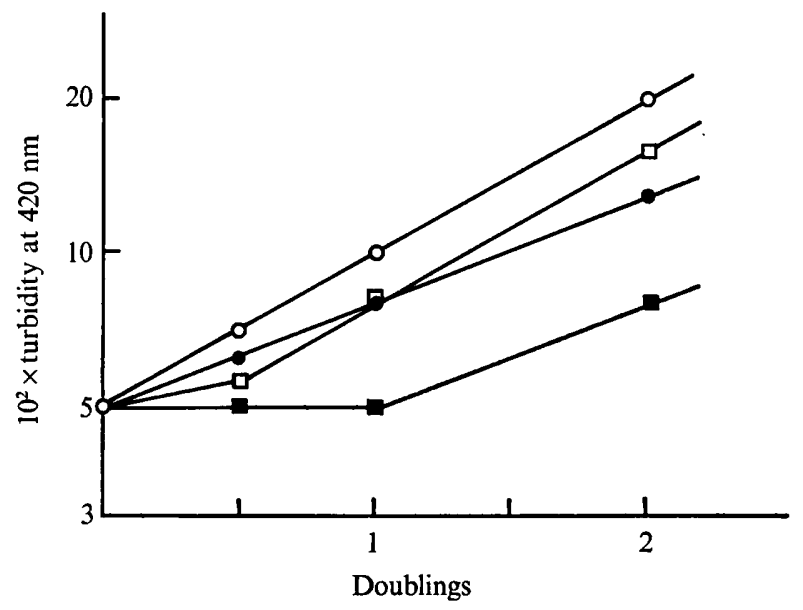

Fig. 2. Effect of a change in carbon source on the growth inhibition of $E$. coli KI 2/I 54 by nonanoic acid. Mid-exponential-phase cultures in succinate medium were used as the inoculum for the new media. The dilution was $\mathrm{I}: 25$. The curves from different media were normalized to allow visual comparisons. One doubling time means the time which would be taken for a culture without fatty acid addition to undergo a doubling, whatever the medium. The control curves (open symbols) were theoretically determined from the doubling times of the bacterium in different media and in the absence of fatty acids (for values in minutes see footnote *, Table 1 ). $\bigcirc$, Succinate; $\bigcirc$, succinate $+0.1 \%$ nonanoic acid; $\square$, acetate; $\square$, acetate $+0.1 \%$ nonanoic acid.

medium with or without $0.1 \%$ nonanoic acid. Nonanoic acid inhibited growth only in the acetate medium (Fig. 2). When washed bacteria were used as inoculum the lag phase was extended from $\mathrm{I}$ to about 4 doubling times.

\section{Fatty acid inhibition in different culture media}

The effect of 0.1 and $0.4 \%$ saturated fatty acids ( $\mathrm{C}_{4}$ to $\left.\mathrm{Cr} 6\right)$ and oleic acid on exponentiallygrowing $E$. coli $\mathrm{KI} 2 / \mathrm{I} 54$ was tested in five different culture media. The effects of $0.1 \%$ fatty acids are summarized in Table $\mathrm{I} . \mathrm{C}_{4}$ to $\mathrm{Cr} 6$ acids failed to inhibit bacterial growth at this concentration (an exception being the addition of $\mathrm{C} 8$ to acetate medium). The doubling 
Table I. Change of doubling time of E. coli $\mathrm{KI} 2 / \mathrm{I} 54$ after the addition of $0 \cdot \mathrm{I} \%$ fatty acids

A $0.1 \%$ fatty acid concentration is equivalent to the following contentrations (mM): $\mathrm{C} 4, \mathrm{II}_{4} 4$;

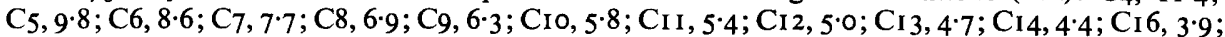
oleic acid, 3.5 . The $0.1 \%$ fatty acids were added to cultures in exponential phase $(E=0.100)$.

Inhibition in different culture media*

\begin{tabular}{cccccc}
\cline { 2 - 5 } Fatty acid addition & Glucose & Acetate & $\begin{array}{c}\text { Nutrient } \\
\text { broth }\end{array}$ & Succinate & $\begin{array}{c}\text { Casamino } \\
\text { acids }\end{array}$ \\
C4 & 47 & 2 & 0 & 16 & II \\
C5 & 72 & 14 & 14 & 12 & -2 \\
C6 & 60 & 18 & 14 & 17 & 20 \\
C7 & 57 & 13 & 14 & 96 & 70 \\
C8 & 37 & $10 \dagger$ & 14 & 72 & 22 \\
C9 & 16 & 6 & 8 & 14 & 25 \\
CI0 & 10 & 17 & 8 & 17 & 26 \\
CII & 10 & 11 & 8 & -2 & 21 \\
CI2 & 10 & 6 & 16 & 11 & 12 \\
CI3 & 0 & -9 & 16 & 0 & 22 \\
CI4 & 2 & -16 & 16 & -16 & I \\
CI6 & -3 & -12 & 16 & -5 & -10 \\
Oleic acid & -3 & -25 & 10 & -3 & 0
\end{tabular}

* Values of inhibition are expressed as: $10^{2} \times$ (doubling time in presence of fatty acid minus doubling time of control culture)/doubling time of control culture. Doubling times for control cultures were (min): glucose, I00; acetate, 420; nutrient broth, 30; succinate, I80; Casamino acids, I35. The data are the average of at least two independent experiments, which did not differ by more than $10 \%$.

$\dagger$ An inhibition phase of 4 to $6 \mathrm{~h}$ was observed.

time was affected by some fatty acids, depending on the culture medium. The short-chain fatty acids influenced the doubling time only in glucose medium, in which the degree of inhibition decreased with increase in chain length. Cio to Ci 6 and oleic acids had no effect on the doubling time. In acetate and nutrient broth a slight inhibitory effect could be detected with some fatty acids, whereas in succinate and Casamino acid medium only the medium-chain fatty acids gave marked inhibition. The degree of growth inhibition increased with chain length up to $\mathrm{C}_{7}$ and decreased from $\mathrm{C}_{10}$ onwards. Longer-chain acids, particularly oleic acid, seemed to produce a growth stimulation in acetate medium. The shorter generation time with oleic acid (I50 $\mathrm{min}$ ) than with acetate (420 $\mathrm{min}$ ) as the sole carbon source for the bacterium may explain these results.

In all media, the addition of $0.4 \%$ medium-chain fatty acids produced a variable lag phase and sometimes (depending on the chain length of the fatty acids) an increase in the doubling time. The short ( $\mathrm{C}_{4}$ to $\mathrm{C}_{5}$ ) and long-chain fatty acids (from $\mathrm{C}_{1} 2$ onwards) only affected the doubling time and no lag phase was present. Figure 3 shows the growth curves up to two doubling times after the addition of $0.4 \%$ medium-chain fatty acids in different media. The effect of decanoic acid was particularly influenced by the nature of the medium. In succinate and acetate minimal media the addition of this fatty acid not only inhibited the growth for at least two doubling times but also caused an actual decrease in cell concentration, while in glucose-containing medium and nutrient broth the bacteria resumed growth after a short lag phase. After 18 to $48 \mathrm{~h}$ (depending on the media), all cultures reached the stationary phase. Undecanoic acid produced little inhibitory effect in any of the media tested. In Table I and Fig. 3 no alteration in effects between odd-numbered $(2 n+1)$ and even-numbered ( $2 n)$ fatty acids could be observed.

The number of viable cells at half the doubling time after the addition of $0.4 \%$ decanoic 

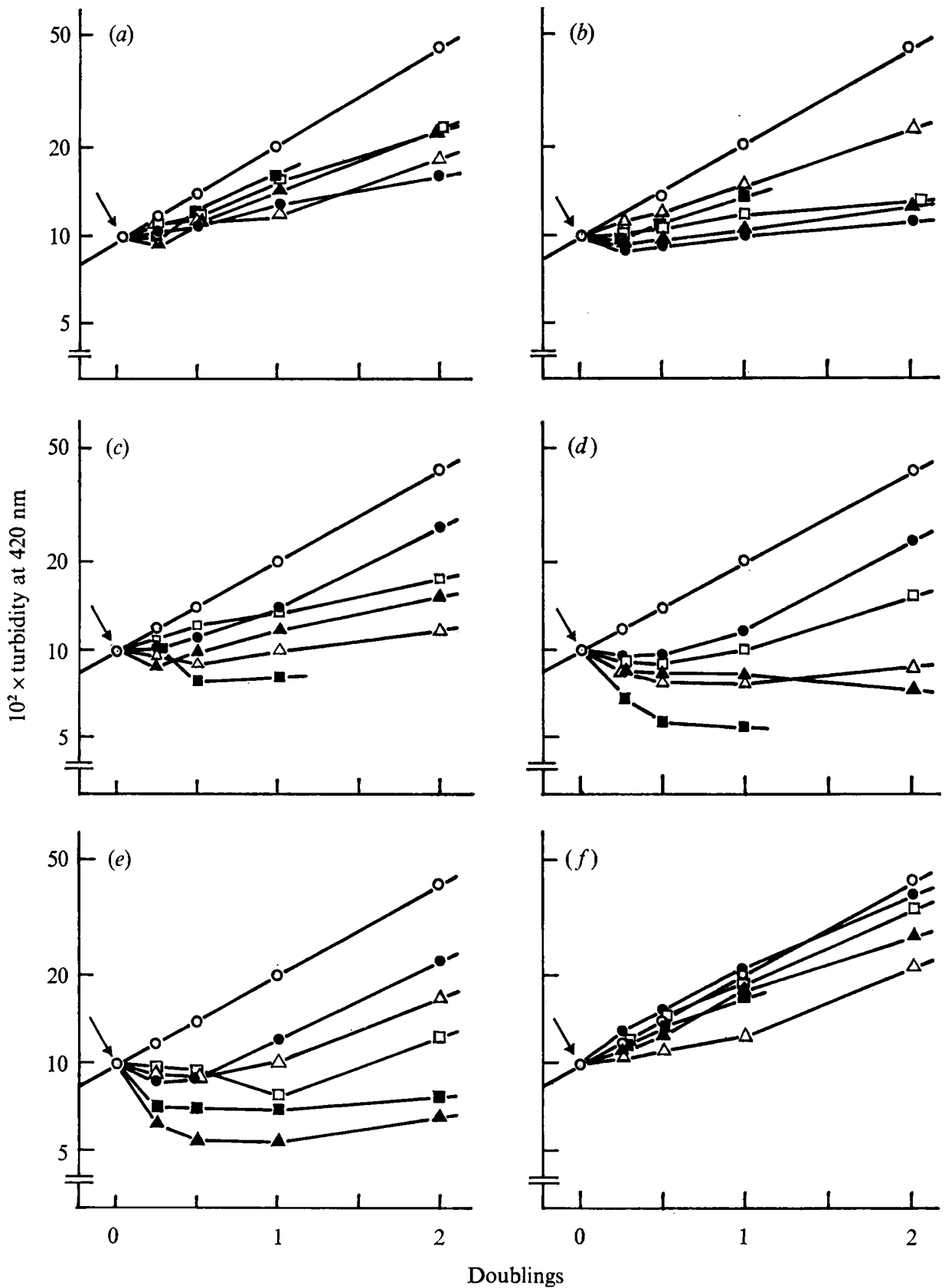

Fig. 3. Effect of $0.4 \%$ medium-chain fatty acids on exponential-phase cultures of $E$. coli KI $2 / 154$ The arrows indicate times of the fatty acid addition: $(a)$ hexanoic $(\mathrm{C} 6) ;(b)$ heptanoic $(\mathrm{C} 7) ;(c)$ octanoic (C8); $(d)$ nonanoic $(\mathrm{C} 9) ;(e)$ decanoic $\left(\mathrm{CI}_{1}\right) ;(f)$ undecanoic $\left(\mathrm{CI}_{1} \mathrm{I}\right)$. The control curves $(\mathrm{O})$ were calculated as in Fig. 2 (for values in minutes see footnote*, Table I). O, Glucose; $\square$, Casamino acids; $\boldsymbol{\square}$, acetate; $\triangle$, nutrient broth; $\boldsymbol{\Delta}$, succinate; $\bigcirc$, control. 
Table 2. Viability remaining after the addition of $0.4 \%$ decanoic acid to E. coli $\mathrm{K} \mathrm{2} 2 / \mathrm{I} 54$ growing exponentially in different media

\begin{abstract}
The viable cell values correspond to the experiments described in Fig. 3. Viability is expressed as the percentage values relative to the number of viable cells before the addition of $0.4 \%$ decanoic acid. The doubling time values in each medium are given in Table $\mathrm{I}$.
\end{abstract}

\begin{tabular}{|c|c|c|c|c|c|}
\hline \multirow[b]{2}{*}{$\begin{array}{l}\text { Viability (measured } \\
\text { after doubling times) }\end{array}$} & \multicolumn{5}{|c|}{ Viable cells $(\%)$, in: } \\
\hline & Glucose & Acetate & $\begin{array}{c}\text { Nutrient } \\
\text { broth }\end{array}$ & Succinate & $\begin{array}{l}\text { Casamino } \\
\text { acids }\end{array}$ \\
\hline 0 & 100 & 100 & 100 & 100 & 100 \\
\hline 0.5 & 60 & 0.2 & 123 & 0.8 & 7 \\
\hline $2 \cdot 0$ & I 82 & 10 & 230 & 3 & I 7 \\
\hline Stationary phase* & + & + & + & + & + \\
\hline
\end{tabular}

* All the cultures reached this phase with a population in the range $1 \times 10^{9}$ to $2 \times 10^{9}$ bacteria $/ \mathrm{ml}$.

acid, showed that the highest bactericidal effect was evident in succinate and acetate media and an intermediate one in Casamino acids medium. After two doubling times the viablecell counts indicated that the bacteria were growing in all media (Table 2).

The killing effect of $0.4 \%$ decanoic acid was increased with stationary-phase cells. In glucose medium, the viable cells remaining at $50 \mathrm{~min}$ after the addition of that acid were $60 \%$ for exponential-phase cultures (Table 2 ) and only I \% for stationary-phase cells.

\title{
Effects of fatty acids on other E. coli strains
}

Some experiments were carried out with $E$. coli strains other than KI $2 / 154$ in an attempt to establish general principles concerning the response of these bacteria to the inhibitory action of fatty acids.

The behaviour of $E$. coli $\mathrm{B}$ and $\mathrm{c}$ cultures growing exponentially in glucose and acetate minimal media or in nutrient broth was studied after the addition of $0.1 \% \mathrm{C}_{4}$ to $\mathrm{C} 16$ and oleic acids. With $E$. coli $\mathrm{B}$ it was found that $\mathrm{C} 6$ and $\mathrm{C} 7$ affected the generation time in all media and $\mathrm{C} 8$ only in nutrient broth. Escherichia coli $\mathrm{C}$ was inhibited by $\mathrm{C} 6$ to $\mathrm{C} 9$ when growing in glucose medium and by $\mathrm{C} 6$ to $\mathrm{C} 8$ in nutrient broth. In acetate minimal medium, the fatty acids from $\mathrm{C} 6$ to $\mathrm{Cr} 6$ produced inhibitory or killing effects on both strains. Although the behaviour of these strains was not identical to that shown by $E$. coli $\mathrm{KI} 2 / \mathrm{I}_{54}$, it provides additional evidence for a correlation between chain length and culture medium and the inhibitory action of fatty acids.

\section{DISCUSSION}

The toxicity of fatty acids towards $E$. coli $\mathrm{K} 12 / 154$ depends on the test conditions used. A given concentration of fatty acid (e.g. $0.1 \%$ ) had different effects, depending on: the chain length of the added fatty acid and culture medium in which the bacteria were grown (Table I); the growth phase of the culture (Fig. I); the presence of an adaptation phase, brought about by changing the culture from one medium to another (Fig. 2); washing of the bacteria. An increase in the fatty acid concentration (e.g. adding $0.4 \% \mathrm{Cro}$ ) produced death of the culture (Table 2).

The experiments described above using $E$. coli strains other than $E$. coli $\mathrm{KI} 2 / \mathrm{I} 54$, indicate that there are many variables that can affect the inhibitory action of fatty acids on $E$. coli. However, some parameters seem to condition a common response in those E. coli strains treated with fatty acids. 
The ability of a fatty acid to induce the enzymes of $\beta$-oxidation and to support growth in $E$. coli is a function of its chain length (Farías \& Trucco, 1972; Klein et al. 1971; Overath, Pauli \& Schairer, 1969; Overath \& Raufuss, 1967; Weeks et al. 1969). The inhibitory effects on $E$. coli were limited, in the majority of cases, to short- and medium-chain fatty acids which are not metabolized by this bacterium (Freese et al. 1973; Salanitro \& Wegener, 1971). The mechanism of inhibition may be related to this fact. The presence of a lipopolysaccharide layer surrounding the walls of Gram-negative organisms could be the reason for the resistance of $E$. coli to long-chain fatty acids (Sheu \& Freese, 1973).

When $0.4 \%$ of a fatty acid was added to cultures growing in the mid-exponential phase in different media, inhibition increased from $\mathrm{C} 6$ to $\mathrm{Cr} 0$ in succinate, acetate and Casamino acids media. With $\mathrm{C}$ Io, the turbidity decrease was found to be related to a decrease in the viable cell counts of the cultures. The addition of a methylene group to decanoic acid (giving undecanoic acid) reversed the inhibition (Fig. 3); this deserves further and more exhaustive studies.

Spector (1946) reported the inhibitory effect of a fatty acid mixture from butter fat and corn oil, on the growth of $E$. coli. Freese et al. (1973) suggested the use of some lipophylic acids as anti-microbial food additives, to replace nitrites and sulphites which can be toxic to man. The variables studied here for bacteriocidal or bacteriostatic actions on $E$. coli cultures by means of fatty acids may be useful in this context.

Escherichia coli $\mathrm{K}$ 2 / I 54was a gift from D. Antón, and E. coli $\mathrm{B}$ and $\mathrm{C}$ strains were obtained through the courtesy of J. P. Bozzini. We thank D. Antón, R. E. Trucco and N. Zwaig for their valuable suggestions and many helpful discussions. This research was supported in part by the Consejo Nacional de Investigaciones Cientifícas y Técnicas de la República Argentina, and forms part of a thesis submitted by J.P.F. to the Universidad Nacional de Tucumàn.

\section{REFERENCES}

AdAms, M. H. (1959). Bacteriophages, p. 446. New York: Interscience.

EISLER, D. M. \& Von MetZ, E. K. (I968). Anti-pasteurella pestis factor. III. Effects of fatty acids on Pasteurella pestis. Journal of Bacteriology 95, 1767-1773.

Farf́as, R. N. \& Trucco, R. E. (1972). Aislamiento y caracterización de mutantes de Escherichia coli que desarrollan en àcidos grasos de cadena corta. Revista Latinoamericana de Microbiología 14, I I-I 3.

Freese, E., Sheu, C. W. \& Galliers, E. (1973). Function of lipophilic acids as antimicrobial food additives. Nature, London 24I, $32 \mathrm{I}-325$.

Kato, A. \& ARIMA, K. (I97I). Inhibitory effect of sucrose ester of lauric acid on the growth of Escherichia coli. Biochemical and Biophysical Research Communications 42, 596-60I.

Klein, K., Steinberg, B., Fiethen, B. \& Overath, P. (197I). Fatty acid degradation in Escherichia coli. An inducible system for the uptake of fatty acids and further characterization of old mutants. European Journal of Biochemistry 19, 442-450.

Nagel de Zwaig, R., Antón, D. N. \& Puig, J. (I962). The genetic control of colicinogenic factors $\mathrm{E}_{2}, \mathrm{I}$ and V. Journal of General Microbiology 29, 473-484.

Overath, P., Pauli, G. \& Schairer, H. U. (1969). Fatty acid degradation in Escherichia coli. An inducible acyl-CoA synthetase, the mapping of the old mutations and the isolation of regulatory mutants. European Journal of Biochemistry 7, 559-574.

Overath, P. \& Raufuss, E. M. (I967). The induction of the enzymes of fatty acid degradation in Escherichia coli. Biochemical and Biophysical Research Communications 29, 28-33.

Salanitro, J. P. \& Wegener, W. S. (I97I). Growth of Escherichia coli on short chain fatty acids. Growth characteristics of mutants. Journal of Bacteriology 108, 885-892.

SHEU, C. W. \& FREESE, E. (1972). Effects of fatty acids on growth and envelope proteins of Bacillus subtilis. Journal of Bacteriology III, 516-524.

SheU, C. W. \& FREeSE, E. (I973). Lipopolysaccharide layer protection of Gram-negative bacteria against inhibition by long chain fatty acids. Journal of Bacteriology $\mathbf{1 1 5}, 869-875$. 
Sheu, C. W., Konings, W. N. \& Freese, E. (1972). Effects of acetate and other short chain fatty acids on sugar and amino acid uptake of Bacillus subtilis. Journal of Bacteriology III, 525-530.

SPECTOR, H. (1946). The comparative effect of the fatty acids of butter fat and corn oil on the growth and metabolism of microorganisms. Archives of Biochemistry II, I67-177.

Weeks, G., Shapiro, M., Burns, R. O. \& WaKIL, S. J. (I969). Control of fatty acid metabolism. I. Induction of the enzymes of fatty acid oxidation in Escherichia coli. Journal of Bacteriology 97, 827-836. 